\title{
Two original experimental setups for Staircase Frontal Affinity Chromatography at the miniaturized scale
}

Andrea Gottardini ${ }^{1}$, Claude Netter ${ }^{2}$, Vincent Dugas ${ }^{1},{ }^{*}$ Claire Demesmay ${ }^{1}$

${ }^{1}$ Université de Lyon, CNRS, Université Claude Bernard Lyon 1, Institut des Sciences Analytiques, UMR 5280, 5 rue de la Doua, F-69100 VILLEURBANNE, France

2 Thermo Fisher Scientific, 16 avenue du Québec, BP 30210, Villebon sur Yvette, FR-91941 Courtabœuf cedex, France

\section{TABLE OF CONTENTS}

UHPLC nano-FAC setup: Protocol detailed description (S1)

Data processing to address non-specific interactions (S2)

\section{LIST OF TABLES}

Table S1. UHPLC tubing dimensions and disposition

\section{LIST OF FIGURES}

Figure S1. Chromatogram results on $\alpha \mathrm{PNG}$

Page S3

Figure S2. Chromatogram results on $\alpha \mathrm{PNGal}$ 


\section{UHPLC nano-FAC setup: Protocol detailed description (S1)}

- $\quad$ (pre-injection phase) 10-way valve moves to position 10-1

- $\quad$ Sample from position GA1 (Buffer) fills loop 1

- $\quad(t=0$ minutes) 10-way valve moves to position 1-2 (Buffer injected in the column)

- $\quad$ Sample from position GA2 (Buffer + DMSO) fills loop 2

- $\quad(t=10$ minutes) 10-way valve moves to position 10-1 (Buffer + DMSO injected in the column, to determine the dead time of the whole setup)

- $\quad$ Sample from position GA1 (Buffer) fills loop 1

- $\quad(\mathrm{t}=20$ minutes) 10-way valve moves to position 1-2 (Buffer rinses column and completes column equilibration)

- $\quad$ Sample from position GA3 (Ligand concentration 1) fills loop 2

- $\quad(t=30$ minutes) 10-way valve moves to position 10-1 (Sample is injected in the column)

At this point, the process continues loading and injecting successively the other ligand concentrations in order, with no column rinse in-between the steps. The time between each step can be modulated based on one's needs and known information about the studied interaction. For the experiments related to this manuscript, each step was 7.5 minutes long, the first concentration being percolated at $\mathrm{t}=30$ minutes, and the last at 75 minutes.

It is worth to note that with $5 \mu \mathrm{L}$ volume and a $300 \mathrm{~nL} / \mathrm{min}$ flow, the percolation can last maximum 15 minutes before risking sample dilution by the buffer liquid phase advancing through the loop. For the experiments carried out for the development of this manuscript, each step was set to be 7.5 minutes long.

\section{Data processing to address non-specific interactions (S2)}

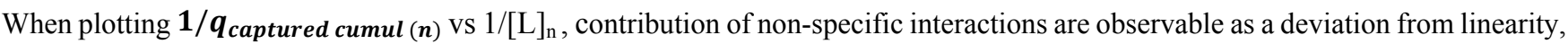
in the high concentration zone of the plot.

To take into account non-specific interactions (if they occur), we must consider that $\boldsymbol{q}_{\text {captured cumul (n) }}$ is equal to sum of the amount of ligand captured by specific interactions $\frac{\boldsymbol{B}_{\text {act }} \times[\boldsymbol{L}]_{n}}{\boldsymbol{K}_{\boldsymbol{d}}+[\boldsymbol{L}]_{n}}$ and of the amount of ligand captured by non-specific interactions $\boldsymbol{K}_{n o n}-$ specific $\times[\boldsymbol{L}]_{\boldsymbol{n}}$. It leads to equation S1:

$q_{\text {captured cumul (n) }}=\frac{B_{a c t} \times[L]_{n}}{K_{d}+[L]_{n}}+K_{\text {non }- \text { specific }} \times[L]_{n}$

(Equation S1)

Where $\mathrm{K}_{\text {non-specific }}$ is a constant related to non-specific interactions ${ }^{1}$.

Equation S1 can be written as follows :

$\frac{1}{q_{\text {captured }}-K_{\text {non-specific }} \times[L]_{n}}=\frac{K_{d}}{B_{\text {act }}} \times \frac{1}{[L]_{n}}+\frac{1}{B_{a c t}}$

(Equation S2)

The excel solver function is used to compute the $\mathrm{K}_{\text {non-specific }}$ factor for which the best $\mathrm{r}^{2}$ value is reached. 
Table S1. UHPLC tubing dimensions and disposition

\begin{tabular}{|c|c|c|c|c|c|c|c|}
\hline & Designation & Connections & & Diameter $[\mu \mathrm{m}]$ & Length $[\mathrm{mm}]$ & Volume $[\mu \mathrm{L}]$ & $\mathrm{P} / \mathrm{N}$ \\
\hline 1 & Connection tube & Inj.valve port 4 & NCS valve port 1 & 20 & 550 & 0.165 & 6041.5260 \\
\hline 2 & Bridge inj. valve & Inj.valve port 2 & Inj.valve port 5 & 20 & 150 & 0.045 & 6041.5121 \\
\hline 3 & Connection tube & NCP pump & Inj.valve port 3 & 20 & 550 & 0.165 & 6041.5260 \\
\hline 4 & Connection tube & Inj.valve port 1 & NCS valve port 4 & 256 & 380 & 18.5 & n.d. \\
\hline 5 & Nano-needle & Inj.valve port 6 & & 125 & 85 & 2.4 & 5820.3010 \\
\hline 6 & Buffer tubing & Siringe valve & NCS valve port 9 & 768 & 620 & 287 & n.d. \\
\hline 7 & Loop 1a (bridge) & NCS valve port 3 & NCS valve port 8 & 100 & 130 & 1 & 6826.2401 \\
\hline 8 & Loop 1 & NCS valve port 2 & NCS valve port 5 & 200 & 160 & 5 & 6826.2405 \\
\hline 9 & Loop 2 & NCS valve port 7 & NCS valve port 10 & 200 & 160 & 5 & 6826.2405 \\
\hline 10 & Column & NCS valve port 6 & $M$ & (20) & As needed & - & - \\
\hline
\end{tabular}

\section{Chromatogram results on $\alpha$ PNG}
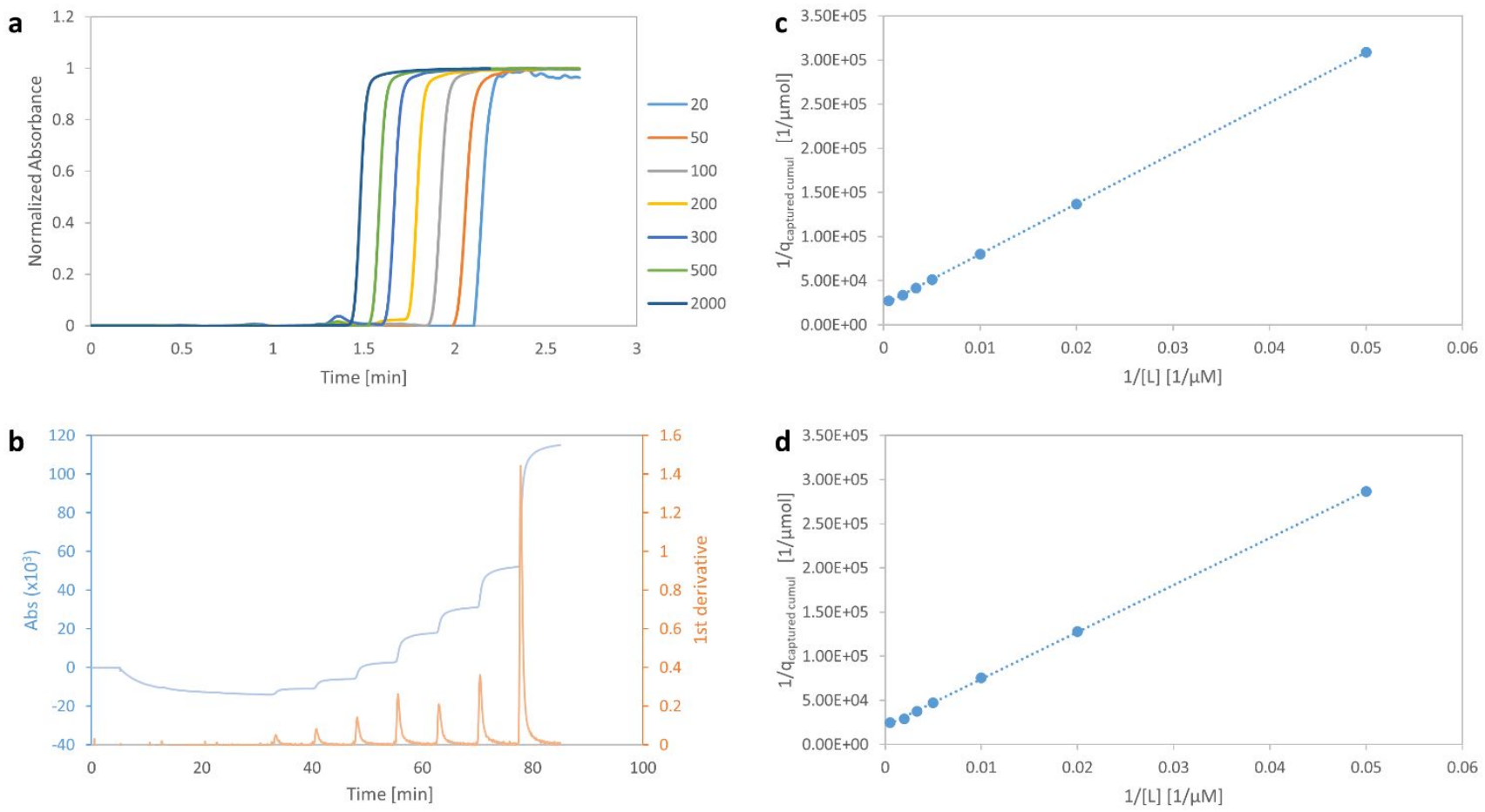

Figure S1. Chromatogram outputs of the staircase nano-FAC analyses carried out on the CE system (a) and the nano-UHPLC system (b)

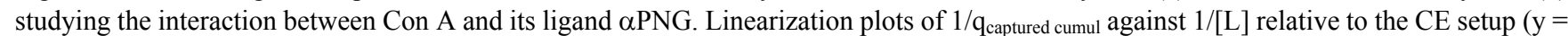
$5709455 \mathrm{x}+22984, \mathrm{r}^{2}=0.99993$, standard deviation of the slope $\mathrm{S}_{\mathrm{b}}=20648$ and standard deviation of the slope $\mathrm{y}$-intercept $\left.\mathrm{S}_{\mathrm{a}}=430\right)(\mathrm{c})$ and UHPLC setup $\left(y=5335708 x+20553, r^{2}=0.99981\right.$, standard deviation of the slope $S_{b}=32800$ and standard deviation of the slope $y-$ intercept $\left.\mathrm{S}_{\mathrm{a}}=684\right)(\mathrm{d})$. 


\section{Chromatogram results on $\alpha$ PNGal}

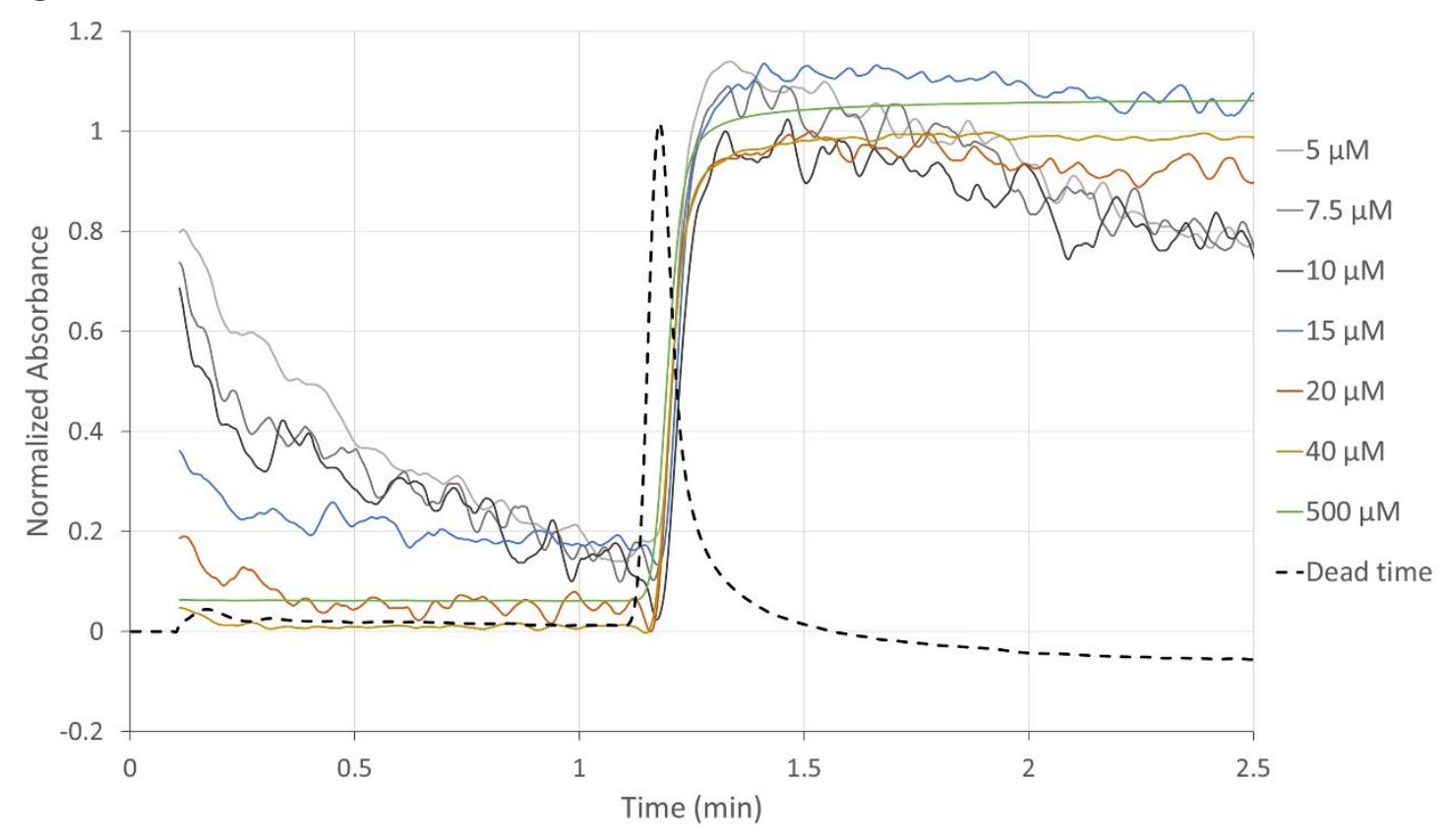

Figure S2. Chromatogram outputs of the staircase nano-FAC analyses carried out on the CE system studying the interaction between Con $\mathrm{A}$ and its ligand $\alpha \mathrm{PNGal}$. All breakthrough curves reach the detector at the dead time as expected for non-binding ligand.

\section{REFERENCES:}

[1] R. Matsuda, Z. Li, X. Zheng, D.S. Hage, J Chromatogr A. 1408 2015, 133-144. 